\title{
Designing of Chain Sampling Plan under Gamma-Poisson Distribution
}

\author{
P. Jeyadurga, S. Balamurali
}

\begin{abstract}
In this manuscript, we discuss the designing procedure of chain sampling plan which is known as one of the conditional sampling plans under gamma-Poisson distribution. We determine the optimal parameters namely, number of items to be chosen for inspection from the lot and number of preceding lots to be considered in order to dispose the current lot by specifying two points on the operating characteristic curve, which is the usual designing approach of sampling plan. The procedure which is used to execute the proposed plan is provided and comparison is made among the proposed plan and existing sampling plans performance.
\end{abstract}

Keywords: chain sampling plan, consumer's risk, discriminating power, gamma-Poisson distribution, producer's risk.

\section{INTRODUCTION} inspection and in which a random sample of items is considered for inspection instead of inspecting all the items. One can reduce the cost and sampling time when implementing the sampling inspection. Acceptance sampling is also sampling inspection which is used for making acceptance or non-acceptance decision on the submitted lot. Many quality professionals use acceptance sampling inspection to provide quality assurance for the manufactured products. Two types of errors are always associated with acceptance sampling, that is, Type I error (or non-acceptance of good quality lot) and Type II error (or acceptance of poor quality lot) since the decision on the lot depends on the information obtained from random sample items. Probabilities of such errors are defined as producer's risk $(\alpha)$ and consumer's risk $(\beta)$ respectively. Acceptable quality level (AQL) and limiting quality level (LQL) are also quality levels correspond to respective producer's risk and consumer's risk. Sampling plans state the sampling rules associated with sampling plans are implemented for both attribute and variables quality characteristic inspection. Count data of sample items (i.e., classification of either conforming or non-conforming to the specifications) is used in attribute

Revised Manuscript Received on December 15, 2019.

* Correspondence Author

P. Jeyadurga, Department of Mathematicss, Kalasalingam Academy of Research and Education, Krishnankoil - 626126, Tamil Nadu, India. Email: jeyadurga.2810@yahoo.com.

S. Balamurali*, Department of Computer Applications, Kalasalingam Academy of Research and Education, Krishnankoil - 626126, Tamil Nadu, India. Email: sbmurali@rediffmail.com.
An alternative method of complete inspection is sampling acceptance criteria for making decision on the lot. Acceptance

quality characteristic inspection whereas the quality measurements are utilized in variables inspection.

Whenever the inspection requires high cost or the product's quality characteristic is of destructive type, the designing of the single sampling plan (SSP) with acceptance number (Ac) either 0 or 1 is preferable. But such sampling plans fail to differentiate the lots whether good or bad (i.e., they have poor discriminating power). In addition, the operating characteristic curve (OC) of SSP with Ac $=0$ has poor shape since the possibility to accept the submitted lot under such sampling plans suddenly decreases even if small change in quality. One can overcome these drawbacks by implementing the sampling plan proposed by [1] namely, chain sampling plan and is denoted as ChSP-1. It is also considered as a conditional sampling plan because it utilizes the previous lots information for the disposition of the current lot. Many authors studied the designing of ChSP-1 under different situations (see for example [2], [3], [4], [5], [6]).

Although the production process is maintained perfectly, there may be some variations in the quality of the products due to random fluctuations. Hence, it can be seen that the quality variation between lots. When quality variation between the lots is higher than that of within lots, the implementation of conventional sampling plans is not appropriate to take a decision on the lot. So, one can use Bayesian sampling plans under this situation and prior information of the process variation can be utilized in such sampling plans. Several authors designed various sampling plans under Bayesian methodology based on gamma-Poisson distribution (see for example, [7], [8], [9]). In addition, Bayesian ChSP-1 has been designed by [4] under gamma-Poisson distribution. But they provided the optimal parameters of Bayesian ChSP-1 based on unity values.

To the best of our knowledge, no one has designed Bayesian ChSP-1 under gamma-Poisson distribution using two points on the OC curve approach. Therefore, in this paper, we try to design such Bayesian ChSP-1 as mentioned above. The configuration of the remaining paper is as follows. The discussion on ChSP-1 under gamma-Poisson distribution is given in Section 2. In Section 3, designing methodology of Bayesian ChSP-1 is discussed for two specified points on the OC curve. In Section 4, we explain the application of the proposed plan and the comparative study is also provided. Some concluding remarks of this study are given in Section 5.

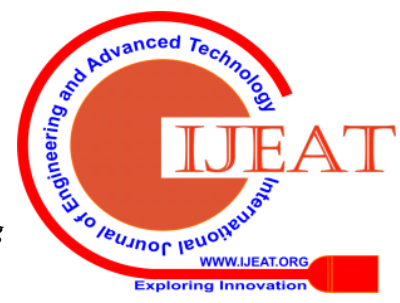




\section{Designing of Chain Sampling Plan underGamma-Poisson Distribution}

\section{CHSP-1 UNDER GAMMA-POISSON DISTRIBUTION}

When we select the sample items from a lot which consists of large number of items or from the process for the inspection, the number of non-conforming items contained in the sample follows a binomial distribution with parameters $n$ and $p$ where $n$ denotes the number of sample items and $p$ denotes the proportion of the non-conforming items. Sometimes, it is suitable to count the number of non-conformities per unit rather than number of items is non-conforming when the samples are taken from the continuous stream of production process. Under this situation, the figure of non-conformities in the sample is modeled by Poisson distribution with parameter $n p$, where $p$ denotes the average number of non-conformities per unit. Then the distribution of figure of non-conforming items (say, $d$ ) is given as follows.

$$
P(d ; x)=\frac{e^{-x}(x)^{d}}{d !}, \text { where } x=n p \text { and } d=0,1,2 \ldots
$$

Since the gamma distribution is a natural conjugate prior to the average number of non-conformities per unit, it will be reasonable that the assumption of $p$ follows gamma distribution when $p$ randomly varies from lot-to-lot. The prior gamma distribution when $p$ randomly varies from lot-to-lot. The prior distribution of $p$ has the following density function.

$$
f(p / a, \delta)=\frac{e^{-a p} a^{\delta} p^{\delta-1}}{\Gamma(\delta)}, 0 \leq p<\infty \text { and } a, \delta>0
$$

where the scale parameter $a=\delta / \bar{p}$ with $E(p)=\bar{p}$, the gamma distribution shape parameter is $\delta$ and the process average is $\bar{p}$. The value of shape parameter $\delta$ is given earlier or it can be estimated from the production process history. The distributions of number of non-conforming items in the sample, $d$ and the average number of non-conformities, $p$ are independent when the manufacturing process is inconsistent. Hence, the sampling distribution of $d$ (see [10]) is given by

$$
\begin{array}{r}
P(d ; n \bar{p}, \delta)=\frac{(\delta+d-1) !}{d !(\delta-1) !}\left(\frac{n \bar{p}}{n \bar{p}+\delta}\right)^{d}\left(\frac{\delta}{n \bar{p}+\delta}\right)^{\delta} \\
d=0,1,2 \ldots
\end{array}
$$

\section{A. Operating Procedure and OC function}

Obviously, ChSP-1 is very stringent and used to inspect the lots which are coming continuously from the production process. The main condition to use this plan is the submitted lot should be the same quality. The proposed ChSP-1 is operated using the following procedure.

1) From current lot, randomly take a sample of $n$ items and count number of non-conforming items is contained in the sample.

2) Accept the current lot if there is no non-conforming sample item and immediately reject the current lot if more than one of the sampled item is non-conforming. If there is one sample item is non-conforming, then accept the current lot provided that none of the preceding ' $i$ ' samples obtained from ' $i$ ' lots contains non-conforming items.
One can characterize ChSP-1 by number of sampled items, $n$ and the parameter $i$, where $i$ denotes the number of preceding lots utilized in making decision on the current lot. The OC function of ChSP-1 (see [1]) is given in the following equation.

$$
P_{a}(p)=P(d=0)+\left\lfloor P(d=1) \times(P(d=0))^{i}\right\rfloor
$$

where probability of current lot acceptance under ChSP-1 is represented by $P_{a}(p), P(d=0)$ represents the probability that there is none of the sample item is non-conforming out of $n$ items and $P(d=1)$ denotes the probability that current sample contains one non-conforming item. The OC function of ChSP-1 under gamma-Poisson distribution is written as in the following manner.

$$
\begin{aligned}
& P_{a}(\bar{p})=\left(\frac{\delta}{n \bar{p}+\delta}\right)^{\delta}+\delta\left(\frac{n \bar{p}}{n \bar{p}+\delta}\right)\left(\frac{\delta}{n \bar{p}+\delta}\right)^{\delta} \\
& {\left[\left(\frac{\delta}{n \bar{p}+\delta}\right)^{\delta}\right]^{\mathrm{i}} }
\end{aligned}
$$

i.e.,

$$
P_{a}(\bar{p})=\left(\frac{\delta}{n \bar{p}+\delta}\right)^{\delta}\left[1+\delta\left(\frac{n \bar{p}}{n \bar{p}+\delta}\right)\left(\frac{\delta}{n \bar{p}+\delta}\right)^{\delta \times i}\right]
$$

The average sample number (ASN) of ChSP-1 is its sample size only. i.e., $\operatorname{ASN}(p)=n$. One can calculate the probability of acceptance of the current lot under proposed ChSP-1 using the above equation for different combinations of $(n ; \delta ; \bar{p})$.

\section{DESIGNING METHODOLOGY}

One of the usual procedures adopted in sampling plan designing is two points on the OC curve approach. This approach admires both producer and consumer since it considers both of their risks. We also prefer the same approach to design the proposed ChSP-1 with the objective of minimizing the ASN. The optimal plan parameters are selected so that which simultaneously satisfy both the risks of producer and consumer and the following optimization problem is used for the purpose of selecting optimal plan with minimum ASN.

$$
\begin{array}{cl}
\text { Minimize } & A S N(p)=n \\
\text { Subject to } & P_{a}\left(p_{1}\right) \geq 1-\alpha \\
& P_{a}\left(p_{2}\right) \leq \beta \\
& n>1, i \geq 1
\end{array}
$$

where

$$
\begin{aligned}
& P_{a}\left(p_{1}\right)=\left(\frac{\delta}{n p_{1}+\delta}\right)^{\delta}\left[1+\delta\left(\frac{n p_{1}}{n p_{1}+\delta}\right)\left(\frac{\delta}{n p_{1}+\delta}\right)^{\delta i}\right] \\
& P_{a}\left(p_{2}\right)=\left(\frac{\delta}{n p_{2}+\delta}\right)^{\delta}\left[1+\delta\left(\frac{n p_{2}}{n p_{2}+\delta}\right)\left(\frac{\delta}{n p_{2}+\delta}\right)^{\delta i}\right]
\end{aligned}
$$

Here $P_{a}\left(p_{1}\right)$ and $P_{a}\left(p_{2}\right)$ denote the acceptance probabilities of the lot where the quality levels are AQL and LQL respectively.

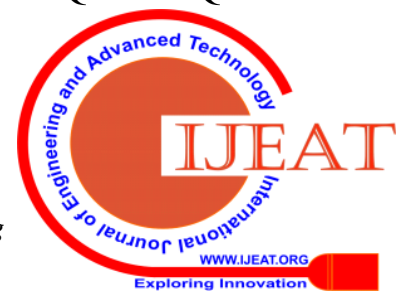


Tables I-III report the optimal values of $n$ and $i$ for three specified values of AQL $\left(=p_{1}\right)$ such as $0.001,0.0025,0.005$ but different combinations of LQL $\left(=p_{2}\right)$. In this designing, it is considered as the value of gamma-Poisson distribution's shape parameter is known and taken as $\delta=5,10,25,50,100$ and $\delta=150$. We determine the optimal parameters so that which satisfy the risk of producer's at $\alpha=0.05$ and the risk of consumer's at $\beta=0.10$ simultaneously. It is obvious from these tables that there is a reduction in sample size if there is an increment either in shape parameter or in the value of $\left(=p_{2}\right)$. There is no certain trend observed when the value of $\left(=p_{1}\right)$ increases and the optimal parameters are relatively same when shape parameter value is increased from 100 to 150.

Table I Optimal parameters of the proposed plan for $\alpha=0.05$ and $\beta=0.10$ when $p_{1}=0.001$

\begin{tabular}{|c|c|c|c|c|}
\hline \multirow{2}{*}{$\begin{array}{c}\text { Shape } \\
\text { parameter } \\
(\delta)\end{array}$} & \multicolumn{4}{|c|}{ Limiting quality level $\left(p_{2}\right)$} \\
\cline { 3 - 5 } & 0.02 & 0.03 & 0.04 & 0.05 \\
\hline 5 & $(148,2)$ & $(99,2)$ & $(74,2)$ & $(60,2)$ \\
10 & $(130,3)$ & $(87,3)$ & $(65,3)$ & $(52,3)$ \\
25 & $(121,3)$ & $(81,3)$ & $(61,2)$ & $(49,2)$ \\
50 & $(118,3)$ & $(79,3)$ & $(59,3)$ & $(48,2)$ \\
100 & $(117,3)$ & $(78,3)$ & $(59,2)$ & $(48,2)$ \\
150 & $(117,3)$ & $(78,3)$ & $(59,2)$ & $(47,2)$ \\
\hline
\end{tabular}

Table II Optimal parameters of the proposed plan for $\alpha=0.05$ and $\beta=0.10$ when $p_{1}=\mathbf{0 . 0 0 2 5}$

\begin{tabular}{|c|c|c|c|c|}
\hline \multirow{2}{*}{$\begin{array}{c}\text { Shape } \\
\text { parameter } \\
(\delta)\end{array}$} & \multicolumn{4}{|c|}{ Limiting quality level $\left(p_{2}\right)$} \\
\cline { 2 - 5 } & 0.06 & 0.07 & 0.08 & 0.09 \\
\hline 5 & & & & \\
10 & $(49,4)$ & $(42,6)$ & $(37,8)$ & $(36,1)$ \\
25 & $(41,2)$ & $(37,8)$ & $(36,1)$ & $(32,1)$ \\
50 & $(40,2)$ & $(34,2)$ & $(31,2)$ & $(28,2)$ \\
100 & $(40,2)$ & $(34,2)$ & $(30,2)$ & $(27,2)$ \\
150 & $(40,2)$ & $(34,2)$ & $(30,2)$ & $(27,2)$ \\
\hline
\end{tabular}

Table III Optimal parameters of the proposed plan for $\alpha=0.05$ and $\beta=0.10$ when $p_{1}=0.005$

\begin{tabular}{|c|c|c|c|c|}
\hline \multirow{2}{*}{$\begin{array}{c}\text { Shape } \\
\text { parameter }\end{array}$} & \multicolumn{4}{|c|}{ Limiting quality level $\left(p_{2}\right)$} \\
\cline { 2 - 5 }$(\delta)$ & 0.07 & 0.08 & 0.09 & 0.10 \\
\hline 5 & $*$ & $(40,1)$ & $(36,1)$ & $(30,2)$ \\
10 & $(41,1)$ & $(36,1)$ & $(30,2)$ & $(26,3)$ \\
25 & $(38,1)$ & $(31,2)$ & $(27,3)$ & $(25,3)$ \\
50 & $(37,1)$ & $(30,2)$ & $(27,3)$ & $(24,4)$ \\
100 & $(36,1)$ & $(30,2)$ & $(26,3)$ & $(24,4)$ \\
150 & $(36,1)$ & $(30,2)$ & $(26,3)$ & $(24,4)$ \\
\hline
\end{tabular}

Note: * Plan does not exist.

\section{APPLICATION OF THE PROPOSED PLAN}

A quality professional wishes to make a decision on the lot and he/she assumes that the products quality characteristic considered in this study follows gamma-Poisson distribution with shape parameter $\delta=10$. The values of AQL, LQL, producer and consumer risks are specified as $\mathrm{AQL}=0.001$, $\mathrm{LQL}=0.05, \alpha=0.05, \beta=0.10$. The quality professional wants that the product quality should be good quality and the decision should be made using minimum number of sample items. Therefore, the proposed ChSP-1 based on gamma-Poisson distribution is suggested to take a decision on the submitted lot since it requires minimum number of sample items and the inspection under proposed plan will be stringent. The optimal parameters obtained from Table I are $n$ $=52, i=3$ to implement the proposed plan and these parameters are corresponding to the above specified requirements. The proposed plan is implemented as follows. Select a sample of 52 items randomly from the current lot and count number of sample items is non-conforming. Accept the current lot if no one sample item is found to be non-conforming and reject the current lot if more than one sample item is non-conforming. If there is one sample item is non-conforming, then accept the current lot provided that preceding 3 lots have been accepted without non-conforming items.

\section{A. Comparative study}

To prove the advantages of the proposed ChSP-1, the comparison between the ASN (or sample size) of the proposed plan and the sample size of SSP proposed by [7] is done. The ASN of both plans for the fixed value of AQL $\left(=p_{1}\right)=0.005$ and three different LQL values, i.e., $p_{2}=0.07$, $0.08,0.09$ are provided in Table IV. Based on the approach of two specified points on the OC curve, we determine these sample sizes for different shape parameters. It is observed from Table IV that the proposed plan's ASN is smaller than the same of SSP. This means that the proposed ChSP-1 will require minimum sample size to dispose the current lot when compared with SSP. For instance, when $p_{2}=0.08, \delta=5$, the proposed ChSP-1 makes an acceptance or rejection decision using 40 sample items but 66 sample items are required under SSP in making a decision. It can be concluded from this discussion that the proposed plan will be very helpful in attaining the acceptance or rejection decision on the current lot using minimum number of sample items rather than SSP with same protection obtained under SSP.

Table IV ASN of the proposed gamma-Poisson ChSP-1 and gamma-Poisson SSP when $p_{1}=0.005$

\begin{tabular}{|c|c|c|c|c|c|c|}
\hline \multirow{3}{*}{\begin{tabular}{c} 
Shape \\
parameter \\
\cline { 2 - 7 }
\end{tabular}} & \multicolumn{6}{|c|}{0.07 Limiting quality level $\left(p_{2}\right)$} \\
\cline { 2 - 7 } & ChSP-1 & SSP & ChSP-1 & SSP & ChSP-1 & SSP \\
\hline 5 & $*$ & 106 & 40 & 66 & 36 & 58 \\
10 & 41 & 65 & 36 & 57 & 30 & 50 \\
25 & 38 & 59 & 31 & 52 & 27 & 46 \\
50 & 37 & 58 & 30 & 51 & 27 & 45 \\
150 & 36 & 57 & 30 & 50 & 26 & 44 \\
\hline
\end{tabular}

Note: * Plan does not exist.

Obviously, one can study that how the sampling plan discriminates good quality lots and poor quality lots using the OC curve of the plan. So, 


\section{Designing of Chain Sampling Plan underGamma-Poisson Distribution}

we consider the OC curves of proposed plan along with the same of SSP with Ac $=0$ to investigate the performance of the plans. In order to construct the OC curves, we consider the AQL and LQL values such as $p_{1}=0.005, p_{2}=0.08$ and the shape parameter $\delta=5$. Then the OC curve of proposed plan is drawn for $n=40, i=1$ and the same of SSP with Ac $=0$ is drawn for the sample size $n=66$ also they are shown in Figure 1. From this figure, it is observed that the proposed plan provides higher chance to accept the current lot than the chance under SSP with Ac $=0$ if the value of proportion non-conforming is small. That is, the producer protection is assured under the proposed plan when the product has good quality. Similarly, the proposed ChSP-1 yields minimum chance for accepting the current lot than that of SSP with $A c=0$ if there is an increment in proportion non-conforming (i.e., quality decreases). This ensures the consumer protection under proposed plan when the product has poor quality. Therefore, the conclusion made from this discussion that the proposed plan will greatly differentiate the lots with respect to their quality when compared to the discriminating ability of $\mathrm{SSP}$ with $\mathrm{Ac}=0$.

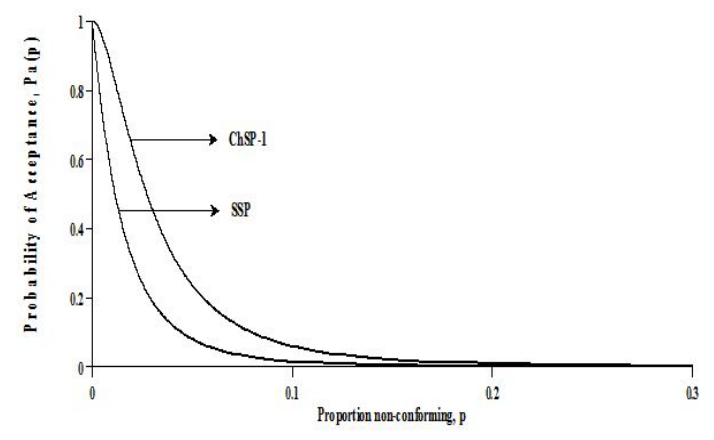

Fig. 1. OC curves of proposed ChSP-1 and SSP with $\mathrm{Ac}=\mathbf{0}$

\section{CONCLUSIONS}

In this paper, we have designed the chain sampling plan under gamma-Poisson distribution by specifying two points on the operating characteristic curve. It has been described the implementation of the proposed sampling plan and the efficiency of the proposed sampling plan in lot sentencing using minimum sample size has been discussed. The comparison of the operating characteristic curves of the proposed plan and single sampling plan with zero acceptance number shows that the discriminating power of the proposed chain sampling plan is better than the same of single sampling plan with zero acceptance number. It is concluded from this study that the producer as well as the consumer can attain the same protection under proposed plan as in zero acceptance number single sampling plan with minimum cost and time.

\section{ACKNOWLEDGMENT}

The authors would like to thank the editor and anonymous reviewers for their precious suggestions and comments which significantly led to improve the presentation of the manuscript. The first author would like to thank the
Kalasalingam Academy of Research and Education for providing financial support through Post-doctoral fellowship.

\section{REFERENCES}

1. H. F. Dodge, "Chain sampling inspection plan", Ind. Qual. Control, vol. 11, 1955, pp. 10-13.

2. V. Soundararajan, "Procedures and tables for construction and selection of chain sampling plans (ChSP-1) - Part I", J. Qual. Technol., vol. 10, 1978, pp. $56-60$

3. K. Govindaraju, and S. Balamurali, "Chain sampling plan for variables inspection”, J. Appl. Stat., vol. 25, 1998, pp. 103-109.

4. R. Vijayaraghavan, and K. M. Sakthivel, "Chain sampling inspection plans based on Bayesian methodology", Econ. Qual. Control, vol. 26, 2011, pp. 173-187.

5. S. Balamurali, "Chain Sampling”, Wiley Stats Ref: Statistics Reference Online, 2016, pp. 1-6.

6. S. Balamurali, P. Jeyadurga, M. Usha, and M. Venkatesulu, "Optimal designing of chain sampling plan for Weibull distributed percentile life assurance", Int. J. Math. Comput., vol. 29, 2018, pp. 94-105.

7. R. Vijayaraghavan, K. Rajagopal, and A. Loganathan, "A procedure for selection of gamma-Poisson single sampling plan by attribute", J. Appl. Stat., vol. 35, 2008, pp. 149-160.

8. M. Aslam, S. Balamurali, C. -H. Jun, M. Ahmad, and M. Rasool, "Designing of group sampling plans based on gamma-Poisson distribution", J. Test. Eval., vol. 40, 2012, pp. 1-6.

9. S. Balamurali, P. Jeyadurga, and M. Usha, "Designing of Bayesian multiple deferred state sampling plan based on gamma-Poisson distribution", Amer. J. Math. Manag. Sci., vol. 35, 2016, pp. 77-90.

10. A. Hald, "Statistical Theory of Sampling Inspection by Attributes", Academic Press, New York, 1981.

\section{AUTHORS PROFILE}

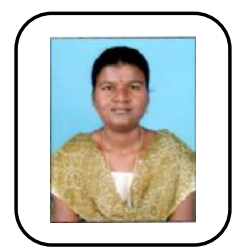

P. Jeyadurga completed her M. Sc. in Mathematics in Madurai Kamaraj University and received her Ph. D., degree from Kalasalingam Academy of Research and Education. Her areas of interest are acceptance sampling, control charts.

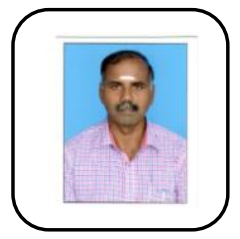

S. Balamurali is a professor of Statistics at Kalasalingam University. He earned his M.Sc. and Ph.D. degrees from Bharathiyar University, India. His research interests include statistical process control, acceptance sampling and analysis of means. 